\title{
AN EXTENDED HAMBURGER MOMENT PROBLEM
}

\author{
by OLAV NJÅSTAD
}

(Received 18th June, 1984)

\section{Introduction}

The classical Hamburger moment problem can be formulated as follows: Given a sequence $\left\{c_{n}: n=0,1,2, \ldots\right\}$ of real numbers, find necessary and sufficient conditions for the existence of a distribution function $\psi$ (i.e. a bounded, real-valued, non-decreasing function) on $(-\infty, \infty)$ with infinitely many points of increase, such that $c_{n}=\int_{-\infty}^{\infty} t^{n} d \psi(t)$, $n=0,1,2, \ldots$. This problem was posed and solved by Hamburger [5] in 1921. The corresponding problem for functions $\psi$ on the interval $[0, \infty)$ had already been treated by Stieltjes [15] in 1894. The characterizations were in terms of positivity of Hankel determinants associated with the sequence $\left\{c_{n}\right\}$, and the original proofs rested on the theory of continued fractions. Much work has since been done on questions connected with these problems, using orthogonal functions and extension of positive definite functionals associated with the sequence. Accounts of the classical moment problems with later developments can be found in $[1,4,14]$. Good modern accounts of the theory of orthogonal polynomials can be found in $[2,3]$.

In 1980 Jones, Thron and Waadeland [6,11] introduced the strong moment problems. The strong Hamburger moment problem (SHMP) may be formulated as follows: Given a double sequence $\left\{c_{n}: n=0, \pm 1, \pm 2, \ldots\right\}$ of real numbers, find necessary and sufficient conditions for the existence of a distribution function $\psi$ on $(-\infty, \infty)$ with infinitely many points of increase, such that $c_{n}=\int_{-\infty}^{\infty} t^{n} d \psi(t), n=0, \pm 1, \pm 2, \ldots$. The corresponding problem for the case that $\psi$ is a function on the interval $[0, \infty)$ was solved in [11], the theory of continued fractions being used in the proofs. The SHMP was solved for a restricted class of sequences in [9], again by the use of continued fractions. A solution in the general case was given in [10], where the theory of orthogonal Laurent polynomials ( $L$-polynomials) introduced in [6] was used as the main tool in the proofs. The characterizations were again given in terms of positivity of Hankel determinants associated with $\left\{c_{n}\right\}$, or alternatively in terms of positive definiteness of a functional associated with the sequence. An exposition of the theory of orthogonal $L$-polynomials is given in [12]. Discussions of orthogonal $L$-polynomials and the strong moment problems can also be found in $[7,8,13]$.

In this paper we discuss the following extended Hamburger moment problem (EHMP) defined as follows: Given $p$ real numbers $a_{1}, \ldots, a_{p}$ and $p$ sequences $\left\{c_{n}^{(r)}: n=1,2, \ldots\right\}$, $r=1,2, \ldots, p$, of real numbers, find necessary and sufficient conditions for the existence of a distribution function $\psi$ on $(-\infty, \infty)$ with infinitely many points of increase, such that

$$
\int_{-\infty}^{\infty} d \psi(t)=1, \quad c_{n}^{(r)}=\int_{-\infty}^{\infty} \frac{d \psi(t)}{\left(t-a_{r}\right)^{n}}, \quad r=1, \ldots, p, \quad n=1,2, \ldots .
$$


The solution is given in terms of positive definiteness of a functional $\phi$ associated with the given sequences. The main tool used in the proofs is the theory of orthogonal $R$-functions. $R$-functions are functions of the form

$$
\alpha_{0}+\sum_{r=1}^{p} \sum_{j=1}^{N_{r}} \frac{\alpha_{r j}}{\left(t-a_{r}\right)^{j}}
$$

Our treatment of the EHMP is closely related to those of the classical and strong moment problems that use orthogonal (and quasi-orthogonal) function systems as important tools. In the course of the exposition we prove a few results on orthogonal $R$-functions that are not strictly needed for the solution of the EHMP, but which may be of some interest in themselves.

We also discuss to some extent quasi-orthogonal $R$-functions. Some results on orthogonal $R$-functions are special cases of results on quasi-orthogonal $R$-functions, and can be easily obtained from these. We have, however, preferred to give direct proofs for all results on orthogonal $R$-functions.

\section{Preliminaries}

1.1. Let $a_{1}, \ldots, a_{p}$ be given (fixed) real numbers, Let $\mathscr{R}$ denote the linear space consisting of all functions of the form

$$
R(t)=\alpha_{0}+\sum_{r=1}^{p} \sum_{j=1}^{N_{r}} \frac{\alpha_{r j}}{\left(t-a_{r}\right)^{j}}, \alpha_{0}, \alpha_{i j} \in \mathbb{R} .
$$

Elements of $\mathscr{R}$ shall be called $R$-functions. (We may also consider complex $R$-functions, i.e. functions where the coefficients $\alpha_{0}, \alpha_{r j}$ are complex numbers. The results in Section 1 are valid for complex $R$-functions.)

We note that a function $R(t)$ belongs to $\mathscr{R}$ iff it can be written in the form $R(t)=$ $P(t) / Q(t)$, where $Q(t)$ is a polynomial with all its zeros among the points $a_{1}, \ldots, a_{p}$, and where $P(t)$ is a polynomial such that $\operatorname{deg} P \leqq \operatorname{deg} Q(\operatorname{deg} P$ denotes the degree of the polynomial $P(t)$ ). This follows by partial fraction decomposition. Thus the $R$-functions are exactly the rational functions whose only poles in the extended complex plane are among the points $a_{1}, \ldots, a_{p}$. Clearly the space $\mathscr{R}$ is closed under multiplication.

1.2. The $L$-polynomials discussed e.g. in $[10,12]$ are of the form $L(t)=\sum_{j=m}^{n} \alpha_{j} t^{j}$. They are not $R$-functions, but are related to $R$-functions in the sense that they correspond to the points $a_{1}, \ldots, a_{p}$ being replaced by $0, \infty$. Ordinary polynomials correspond to the one distinguished point $\infty$.

1.3. We write $\mathscr{R}\left(s_{1}, s_{2}, \ldots, s_{p}\right)$ for the space of all $R$-functions

$$
R(t)=\frac{P(t)}{\left(t-a_{1}\right)^{s_{1}}\left(t-a_{2}\right)^{s_{2}} \ldots\left(t-a_{p}\right)^{s_{p}}}, \operatorname{deg} P \leqq s_{1}+s_{2}+\cdots+s_{p} .
$$


Every natural number $n$ has a unique decomposition $n=p \cdot q_{n}+r_{n}, 1 \leqq r_{n} \leqq p$. We shall write $n=p q+r$ when there is no danger of confusion. We shall write $\mathscr{R}_{n}$ for $\mathscr{R}\left(s_{1}, \ldots, s_{p}\right)$ in the special case that $s_{1}=\cdots=s_{r}=q+1, s_{r+1}=\cdots=s_{p}=q$. Thus an element of $\mathscr{R}_{n}$ may by written

$$
R(t)=\frac{P(t)}{\left(t-a_{1}\right)^{q+1} \ldots\left(t-a_{r}\right)^{q+1} \ldots\left(t-a_{p}\right)^{q}}
$$

where $\operatorname{deg} P \leqq n$. We shall denote by $\mathscr{R}^{0}$ (resp. $\left.\mathscr{R}_{n}^{0}, \mathscr{R}^{0}\left(s_{1}, \ldots, s_{p}\right)\right)$ the subspace of those $R$-functions in $\mathscr{R}$ (resp. $\mathscr{R}_{n}, \mathscr{R}\left(s_{1}, \ldots, s_{p}\right)$ ) where $\operatorname{deg} P<n$, i.e. those functions for which $\alpha_{0}=0$.

A base for the space $\mathscr{R}$ is given by

$$
\mathscr{B} \sim\left\{1, \frac{1}{\left(t-a_{1}\right)}, \ldots, \frac{1}{\left(t-a_{p}\right)}, \frac{1}{\left(t-a_{1}\right)^{2}}, \ldots, \frac{1}{\left(t-a_{p}\right)^{2}}, \frac{1}{\left(t-a_{1}\right)^{3}}, \ldots\right\} .
$$

The initial section

$$
\mathscr{B}_{n}=\left\{1, \frac{1}{\left(t-a_{1}\right)}, \ldots, \frac{1}{\left(t-a_{p}\right)}, \frac{1}{\left(t-a_{1}\right)^{2}}, \ldots, \frac{1}{\left(t-a_{r}\right)^{q+1}}\right\}
$$

is a base for the subspace $\mathscr{R}_{n}$.

\section{Orthogonal $R$-functions}

2.1. In the following, $\phi$ shall denote a (fixed) linear functional on $\mathscr{R}$. This functional gives rise to a bilinear form $\langle$,$\rangle on \mathscr{R} \times \mathscr{R}$, defined by $\langle A, B\rangle=\phi(A \cdot B)$. We shall assume that $\phi$, and hence $\langle$,$\rangle is positive definite, i.e. that \phi\left(R^{2}\right)>0$ when $R \in \mathscr{R}, R(t) \equiv 0$. We formally state as a lemma the following easy result.

Lemma 2.2. Let $R \in \mathscr{R}, R(t) \geqq 0$ for all $t \in(-\infty, \infty), R(t) \not 0$. Then $\phi(R)>0$.

Proof. The $R$-function $R(t)$ can be written in the form

$$
R(t)=\frac{A(t)}{C(t)^{2}}
$$

where $C(t)=\left(t-a_{1}\right)^{s_{1}} \ldots\left(t-a_{p}\right)^{s_{p}}$ and $A$ is a non-negative polynomial such that $\operatorname{deg} A \leqq 2 \operatorname{deg} C$. It is well known that every non-negative polynomial may be written as a sum of two squared polynomials (see e.g. [4]). So there are polynomials $D$ and $E$ such that $A(t)=D(t)^{2}+E(t)^{2}$. It follows that

$$
R(t)=\left(\frac{D(t)}{C(t)}\right)^{2}+\left(\frac{E(t)}{C(t)}\right)^{2}
$$


where clearly $\operatorname{deg} D \leqq \operatorname{deg} C, \operatorname{deg} E \leqq \operatorname{deg} C$. Thus $D / C \in \mathscr{R}, E / C \in \mathscr{R}$, and

$$
\phi(R)=\phi\left(\left(\frac{D}{C}\right)^{2}\right)+\phi\left(\left(\frac{E}{C}\right)^{2}\right)>0
$$

2.3. By applying the Gram-Schmidt orthonormalization process (with respect to the inner product defined above) to the sequence

$$
\left\{1, \frac{1}{\left(t-a_{1}\right)}, \ldots, \frac{1}{\left(t-a_{p}\right)}, \frac{1}{\left(t-a_{1}\right)^{2}}, \ldots, \frac{1}{\left(t-a_{p}\right)^{2}}, \frac{1}{\left(t-a_{1}\right)^{3}}, \ldots\right\}
$$

(in the indicated order) we obtain an orthonormal sequence $\left\{Q_{n}: n=0,1,2, \ldots\right\}$ of $R$-functions. Since $Q_{n} \in \mathscr{R}_{n}-\mathscr{R}_{n-1}$ we may write

$$
Q_{n}(t)=\beta_{0}+\sum_{i=1}^{r} \sum_{j=1}^{q+1} \frac{\beta_{i j}}{\left(t-a_{i}\right)^{j}}+\sum_{i=r+1}^{p} \sum_{j=1}^{q} \frac{\beta_{i j}}{\left(t-a_{i}\right)^{j}},
$$

where $r=r_{n}, q=q_{n}, \beta_{r, q+1} \neq 0$. It follows that we may also write

$$
Q_{n}(t)=\frac{B_{n}(t)}{\left(t-a_{1}\right)^{q+1} \ldots\left(t-a_{r}\right)^{q+1}\left(t-a_{r+1}\right)^{q} \ldots\left(t-a_{p}\right)^{q}},
$$

where $B_{n}$ is a polynomial of degree at most $n$, and $B_{n}\left(a_{r}\right) \neq 0$. (Note that here and in the following $a_{r+1}$ means $a_{1}$ if $r=p$, and $a_{r-1}$ means $a_{p}$ if $r=1$.)

Proposition 2.4. The polynomial $B_{n}$ has degree at least equal to $n-1$, and all its zeros are real and simple.

Proof. Let $t_{1}, \ldots, t_{\lambda}$ be all the real zeros of $B_{n}$ of odd order. Assume that $\lambda<n-1$, and set

$$
T(t)=\frac{\left(t-t_{1}\right) \ldots\left(t-t_{\lambda}\right)}{\left(t-a_{1}\right)^{q+1} \ldots\left(t-a_{r-1}\right)^{q+1}\left(t-a_{r}\right)^{q-1}\left(t-a_{r+1}\right)^{q} \ldots\left(t-a_{p}\right)^{q}} .
$$

Then $T \in \mathscr{R}_{n-1}$, since the denominator has degree $n-2$. Furthermore $T \cdot Q_{n}$ is a product of squares and a non-negative (or non-positive) factor. This implies $\phi\left(T \cdot Q_{n}\right) \neq 0$, by Lemma 2.2 , since $T \cdot Q_{n}$ is an $R$-function. Consequently $T \notin \mathscr{R}_{n-1}$, which is a contradiction. Thus $\lambda \geqq n-1$, and hence $\operatorname{deg} B_{n} \geqq n-1$. If $\operatorname{deg} B_{n}=n-1$, then $\lambda=n-1$, and so all the zeros are real and simple. If $\operatorname{deg} B_{n}=n$, then at least $n-1$ zeros are real and simple and consequently all the zeros are real and simple.

2.5. We observe that since $Q_{n} \in \mathscr{R}_{n}-\mathscr{R}_{n-1}$, the point $a_{r}$ cannot be a zero of $B_{n}$. The points $a_{i}, i \neq r$, may be zeros of $B_{n}$, though only simple ones. Let $\mu=\mu_{n}$ denote the number of zeros of $B_{n}$, and $v=v_{n}$ the number of zeros of $Q_{n}$. 
Theorem 2.6. The orthonormal $R$-function $Q_{n}$ can be written

$$
Q_{n}(t)=\frac{C_{n}(t)}{\left(t-a_{1}\right)^{\alpha_{1}} \ldots\left(t-a_{r}\right)^{\alpha_{r}} \ldots\left(t-a_{p}\right)^{\alpha_{p}}}
$$

where

$$
C_{n}(t)=k_{n} \cdot\left(t-t_{1}\right) \ldots\left(t-t_{\mu}\right), \quad t_{j} \neq a_{i}
$$

$i=1, \ldots, p, j=1, \ldots, v$. Here $\kappa_{r}=q+1, q \leqq \kappa_{i} \leqq q+1$ for $i<r, q-1 \leqq \kappa_{i} \leqq q$ for $i>r$. Furthermore $n-1 \leqq \mu \leqq n, \mu-p+1 \leqq \nu \leqq \mu, \kappa_{1}+\cdots+\kappa_{p}=n+v-\mu$.

Proof. The theorem follows by cancellation of common factors in the numerator and denominator in the expression (2.2) for $Q_{n}(t)$, the results of Proposition 2.4 and observations of Section 2.5 being taken into account.

2.7. We shall call the $R$-function $Q_{n}$ and the index $n$ degenerate if the number $\mu$ of zeros of $B_{n}$ is $n-1$. (This situation may also be described by saying that $Q_{n}$ has a zero at infinity.) Similarly we shall call $Q_{n}$ and $n$ defective at $a_{i}$ or $a_{i}$-defective if $a_{i}$ is a zero of $B_{n}$. Thus $Q_{n}$ is $a_{i}$-defective if $\kappa_{i}=q$ when $i<r$, if $\kappa_{i}=q-1$ when $i>r$. Recall that $Q_{n}$ cannot be defective at $a_{r}=a_{r_{n}}$. So in particular there cannot be $p a_{p}$-defective indices in a row, for any $\rho=1, \ldots, p$.

We shall call $Q_{n}$ (and $n$ ) defective if it is $a_{i}$-defective for at least one $i$ and maximally defective if it is $a_{i}$-defective for every $i \neq r$. This means that $\kappa_{i}=q$ for every $i<r, \kappa_{i}=q-1$ for every $i>r$, and $v=\mu-p+1$. We shall call $Q_{n}$ (and $n$ ) singular if it is degenerate or defective, otherwise non-singular.

2.8. There exist $R$-functions playing a role similar to that of quasi-orthogonal polynomials and quasi-orthogonal $L$-polynomials in the theory of orthogonal polynomials and $L$-polynomials. For every index $n$ we shall define the quasiorthogonal $R$-functions $Q_{n}(t, \tau)$ in the following way: For every real number $\tau$ we define

$$
Q_{n}(t, \tau)=Q_{n}(t)-\tau \frac{\left(t-a_{r-1}\right)}{\left(t-a_{r}\right)} Q_{n-1}(t)
$$

Clearly $Q_{n}(t, \tau) \in \mathscr{R}_{n}$. (We shall always consider $Q_{n}(t, \tau)$ as a function of $t$ for fixed $\tau$ when making statements $Q_{n}(t, \tau) \in \mathscr{R}, Q_{n}(t, \tau) \in \mathscr{R}_{n}$, etc.) We may write

$$
Q_{n}(t, \tau)=\frac{B_{n}(t, \tau)}{\left(t-a_{1}\right)^{q+1} \ldots\left(t-a_{r}\right)^{q+1} \ldots\left(t-a_{p}\right)^{q}}
$$

where $B_{n}(t, \tau)$ is a polynomial of degree at most $n$. In the following let $\mu(\tau)$ denote the degree of $B_{n}(t, \tau)$ and $v(\tau)$ the number of zeros of $Q_{n}(t, \tau)$. 
Theorem 2.9. The quasi-orthogonal $R$-function $Q_{n}(t, \tau)$ can be written

$$
Q_{n}(t, \tau)=\frac{C_{n}(t, \tau)}{\left(t-a_{1}\right)^{\kappa_{1}(\tau)} \ldots\left(t-a_{r}\right)^{\alpha_{r}(\tau)} \ldots\left(t-a_{p}\right)^{\kappa_{p}(\tau)}}
$$

where $C_{n}(t, \tau)=k_{n}(\tau)\left(t-t_{1}(\tau)\right) \ldots\left(t-t_{v(\tau)}(\tau)\right), \quad t_{j}(\tau) \neq a_{i}, \quad i=1, \ldots, p, \quad j=1, \ldots, v(\tau) . \quad$ Here $q \leqq \kappa_{i}(\tau) \leqq q+1$ for $i \leqq r, \quad q-1 \leqq \kappa_{i}(\tau) \leqq q$ for $i>r$. Furthermore $n-1 \leqq \mu(\tau) \leqq n$, $\mu(\tau)-p \leqq v(\tau) \leqq p(\tau), \kappa_{1}(\tau)+\cdots+\kappa_{p}(\tau)=n+v(\tau)-\mu(\tau)$.

Proof. Let $t_{1}=t_{1}(\tau), \ldots, t_{\lambda}=t_{\lambda}(\tau)$ be the real zeros of $Q_{n}(t, \tau)$ of odd order. Assume that $\lambda<n-1$. We define the $R$-function $T$ as in formula (2.3), and conclude that $\phi\left(Q_{n}(t, \tau) \cdot T(t)\right) \neq 0$. On the other hand $T \in \mathscr{R}_{n-1}$, hence $\phi\left(Q_{n}(t) \cdot T(t)\right)=0$, and

$$
T(t) \cdot \frac{\left(t-a_{r-1}\right)}{\left(t-a_{r}\right)} \in \mathscr{R}_{n-2},
$$

hence

$$
\phi\left(\frac{\left(t-a_{r-1}\right)}{\left(t-a_{r}\right)} Q_{n-1}(t) \cdot T(t)\right)=\phi\left(Q_{n-1} \cdot \frac{\left(t-a_{r-1}\right)}{\left(t-a_{r}\right)} T^{(t)}\right)=0
$$

This is a contradiction. We conclude as in the proof of Proposition 2.4 that $n-1 \leqq \mu(\tau) \leqq n$, and all the zeros of $B_{n}(t, \tau)$ are real and simple.

Then by cancellation of common factors in the numerator and denominator in the expression (2.6) we obtain the desired result.

2.10. In the same way as for orthogonal $R$-functions we may define the quasiorthogonal $R$-functions $Q_{n}(t, \tau)$ to be degenerate, defective, singular, etc. Note that if $Q_{n-1}(t)$ is not degenerate, then $Q_{n}(t, \tau)$ is degenerate for exactly one value of $\tau$. We also observe that $Q_{n}(t, \tau)$ is $a_{\rho}$-defective for at most one value of $\tau$, for a given $\rho=1, \ldots, p$.

\section{Recursion formulas}

3.1. In this section we shall prove some recursion formulas involving several consecutive orthogonal $R$-functions $Q_{n}$. For comparison we recall the well known threeterm recursion formula for orthogonal polynomials (see e.g. [3]) and the following facts about elements of an orthogonal sequence $\left\{Q_{n}\right\}$ of $L$-polynomials: $Q_{n}$ can always be expressed in terms of the four preceding elements $Q_{n-1}, Q_{n-2}, Q_{n-3}, Q_{n-4}$. If $Q_{n-1}$ is non-singular, then $Q_{n}$ can be expressed in terms of the two preceding elements $Q_{n-1}$, $Q_{n-2}$. If $Q_{n}$ is singular it can be expressed in terms of the preceding element $Q_{n-1}$. For these results, see [12]. In the following, $\left\{Q_{n}\right\}$ denotes the sequence of orthonormal $R$-functions determined by $\phi$.

Theorem 3.2. Assume that $Q_{n-m}$ (where $\left.1 \leqq m \leqq p-1\right)$ is non-defective at $a_{r}=a_{r_{n}}$. Then the following $(p+m)$-term-recursion formula holds: 


$$
Q_{n}(t)=\frac{c}{\left(t-a_{r}\right)} Q_{n-m}(t)+\sum_{j=n-p-m+1}^{n-1} \alpha_{j} Q_{j}(t)
$$

where $c$ and $\alpha_{j}$ are constants.

Proof. We may choose the constant $c$ such that the term

$$
\frac{1}{\left(t-a_{r}\right)^{q+1}}
$$

cancel in the partial fraction decompositions of

$$
Q_{n}(t) \text { and } \frac{c}{\left(t-a_{r}\right)} Q_{n-m}(t)
$$

For this value of $c$ we can write

$$
Q_{n}(t)-\frac{c}{\left(t-a_{r}\right)} Q_{n-m}(t)=\sum_{j=0}^{n-1} \alpha_{j} Q_{j}(t)
$$

Let $j \leqq n-p-m$. Then

$$
Q_{f}(t) \in \mathscr{R}_{n-1}, \frac{Q_{j}(t)}{\left(t-a_{r}\right)} \in \mathscr{R}_{n-m-1},
$$

and hence

$$
\alpha_{j}=\left\langle Q_{n}, Q_{j}\right\rangle-c\left\langle\frac{Q_{n-m}(t)}{\left(t-a_{r}\right)}, Q_{j}(t)\right\rangle=\phi\left(Q_{n} \cdot Q_{j}\right)-c \phi\left(Q_{n-m}(t) \cdot \frac{Q_{j}(t)}{\left(t-a_{r}\right)}\right)=0
$$

From this the desired formula immediately follows.

Corollary 3.3. Assume that $Q_{n-1}$ is non-defective at $a_{r}$. Then the following $(p+1)$-term recursion formula holds:

$$
Q_{n}(t)=\frac{c}{\left(t-a_{r}\right)} Q_{n-1}(t)+\sum_{j=n-p}^{n-1} \alpha_{j} Q_{j}(t)
$$

where $c$ and $\alpha_{j}$ are constants.

Proof. Immediate from Theorem 3.2.

3.4. Theorem 3.2 shows that when $Q_{n-m}$ is non-defective at $a_{r}, 1 \leqq m \leqq p-1$, then $Q_{n}$ 
satisfies a $(p+m)$-term recursion formula. The shortest possible such formula is a $(p+1)$ term formula as given in Corollary 3.3. We now show that $Q_{n}$ always satisfies a $(2 p+1)$ term recursion formula.

Theorem 3.5. For all $n$ the following $(2 p+1)$-term recursion formula holds:

$$
Q_{n}(t)=\frac{c}{\left(t-a_{r}\right)} Q_{n-p}(t)+\sum_{j=n-2 p}^{n-1} \alpha_{j} Q_{j}(t)
$$

where $c$ and $\alpha_{j}$ are constants.

Proof. As in the proof of Theorem 3.2 we may choose a constant $c$ such that

$$
Q_{n}(t)-\frac{c}{\left(t-a_{r}\right)} Q_{n-p}(t)
$$

can be written as

$$
\sum_{j=0}^{n-1} \alpha_{j} Q_{j}(t)
$$

Then

$$
Q_{j}(t) \in \mathscr{R}_{n-1}, \frac{Q_{j}(t)}{\left(t-a_{r}\right)} \in \mathscr{R}_{n-p-1} \quad \text { when } \quad j \leqq n-2 p-1
$$

Hence

$$
\begin{aligned}
\alpha_{j} & =\left\langle Q_{n}, Q_{j}\right\rangle-c\left\langle\frac{Q_{n-p}(t)}{\left(t-a_{r}\right)}, Q_{j}(t)\right\rangle \\
& =\phi\left(Q_{n} \cdot Q_{j}\right)-c \phi\left(Q_{n-p}(t) \cdot \frac{Q_{j}(t)}{\left(t-a_{r}\right)}\right)=0 \quad \text { for } j \leqq n-2 p-1 .
\end{aligned}
$$

From this the formula follows.

Proposition 3.6. Every segment $\left\{Q_{s}, Q_{s+1}, \ldots, Q_{s+2 p}\right\}$ contains at least two nondegenerate elements $Q_{n}$.

Proof. The segment $\left\{Q_{0}, Q_{1}, \ldots, Q_{2 p}\right\}$ contains $Q_{0}$ which is non-degenerate, and we conclude from Theorem 3.5 that it therefore must contain at least one more nondegenerate $Q_{n}$. The result now follows by induction on $s$, Theorem 3.5 being used at every step.

Corollary 3.7. There is an infinite number of indices $n$ for which $Q_{n}$ is non-degenerate.

Proof. Immediate. 
Proposition 3.8. Assume that $Q_{n}$ is defective at $a_{i_{1}}, \ldots, a_{i_{\lambda}}$ (and possibly at other points.) Then we may write

$$
Q_{n}(t)=\frac{1}{\left(t-a_{r}\right)}\left(t-a_{i_{1}}\right) \ldots\left(t-a_{i_{\lambda}}\right) \cdot \sum_{j=n}^{n-1} \alpha_{j+1} Q_{j}(t)
$$

where $\alpha_{j}$ are constants.

Proof. We observe that

$$
R(t)=\frac{\left(t-a_{r}\right) Q_{n}(t)}{\left(t-a_{i_{1}}\right) \ldots\left(t-a_{i_{\lambda}}\right)} \in \mathscr{R}_{n-1}
$$

(recall that $a_{i_{1}}, \ldots, a_{i_{\lambda}} \neq a_{r}$ ). Therefore we may write

$$
R(t)=\sum_{j=0}^{n-1} \alpha_{j} Q_{j}(t)
$$

We also observe that

$$
\frac{\left(t-a_{r}\right) Q_{j}(t)}{\left(t-a_{i_{1}}\right) \ldots\left(t-a_{i_{\lambda}}\right)} \in \mathscr{R}_{j+p-1}
$$

(note that if $j=n-p$ then $i_{1}, \ldots, i_{\lambda} \neq r_{n}=r_{j}$ ). Hence

$$
\left\langle R, Q_{j}\right\rangle=\phi\left(\frac{\left(t-a_{r}\right) Q_{f}(t)}{\left(t-a_{i_{1}}\right) \ldots\left(t-a_{i_{2}}\right)} \cdot Q_{n}(t)\right)=0
$$

for $j \leqq n-p$ (i.e. $\left.\mathscr{R}_{j+p-1} \subset \mathscr{R}_{n-1}\right)$.

Consequently

$$
R(t)=\sum_{j=n}^{n-1} \alpha_{j} Q_{j}(t)
$$

from which the desired results follows.

Proposition 3.9. If $Q_{n-p+1}, \ldots, Q_{n-1}$ are maximally defective, then $Q_{n}$ is non-defective.

Proof. If $Q_{n-p+1}, \ldots, Q_{n}$ are all maximally defective, then any sum $\sum_{j=n-p+1}^{n-1} \alpha_{j} Q_{j}$ can be written as $\left(t-a_{r}\right) G(t)$, where $G \in \mathscr{R}_{n-1}$. This makes it impossible for any expression

$$
\frac{\left(t-a_{i_{1}}\right) \ldots\left(t-a_{i_{1}}\right)}{\left(t-a_{r}\right)} \sum_{j=n-p+1}^{n-1} \alpha_{j} Q_{j}(t)
$$


to have a term

$$
\frac{1}{\left(t-a_{r}\right)^{a_{n}+1}}
$$

in its partial fraction decomposition. Hence no formula of the form (3.4) can hold.

Corollary 3.10. Every segment $\left\{Q_{s+1}, \ldots, Q_{s+p}\right\}$ contains at least one $Q_{n}$ which is not maximally defective.

Proof. Immediate.

\section{Quadrature formulas}

4.1. As before let $t_{1}, \ldots, t_{v}$ denote the zeros of $Q_{n}$. We define the fundamental $R$-functions $L_{n, i}, i=1,2, \ldots, v$, by

$$
L_{n, i}(t)=\frac{Q_{n}(t) \cdot\left(t-a_{r}\right)}{Q_{n}^{\prime}\left(t_{i}\right)\left(t-t_{i}\right)\left(t_{i}-a_{r}\right)}
$$

We immediately observe that $L_{n, i} \in \mathscr{R}_{n-1}$ and that $L_{n, i}\left(t_{j}\right)=\delta_{i j}$. Note that $L_{n, i}$ is degenerate iff $Q_{n}$ is degenerate. We shall write $\lambda_{n, i}$ for $\phi\left(L_{n, i}\right)$.

Let $\kappa_{1}, \ldots, \kappa_{p}$ have the same meaning as in Section 2. (cf. Theorem 2.6). We also recall the meaning of $\mathscr{R}\left(s_{1}, \ldots, s_{p}\right)$ and $\mathscr{R}^{0}\left(s_{1}, \ldots, s_{p}\right)$ from Section 1.

Let $f$ be a given function. We define the $n$th interpolating $R$-function $F$ by $F(t)=\sum_{i=1}^{v} f\left(t_{i}\right) L_{n, i}(t)$. Then $F \in \mathscr{R}_{n-1}$ and $F\left(t_{j}\right)=f\left(t_{j}\right), j=1, \ldots, v$.

Theorem 4.2. When $Q_{n}$ is non-degenerate, the quadrature formula

$$
\phi(f)=\sum_{i=1}^{v} \lambda_{n, i} f\left(t_{i}\right)
$$

is valid for every $f \in \mathscr{R}\left(s_{1}, \ldots, s_{p}\right)$, where $s_{1}=\kappa_{1}+q+1, \ldots, s_{r-1}=\kappa_{r-1}+q+1, s_{r}=2 q+1$, $s_{r+1}=\kappa_{r+1}+q, \ldots, s_{p}=\kappa_{p}+q$. When $Q_{n}$ is degenerate, the same formula is valid for $f \in \mathscr{R}^{0}\left(s_{1}, \ldots, s_{p}\right)$.

Proof. Let $f \in \mathscr{R}\left(s_{1}, \ldots, s_{p}\right)$. The function $g=f-F$ (where $F$ is defined as in Section 4.1) also belongs to $\mathscr{R}\left(s_{1}, \ldots, s_{p}\right)$. We note that $g$ is degenerate if both $Q_{n}$ and $f$ are degenerate. Since $g\left(t_{i}\right)=0, i=1,2, \ldots, v$, we may write

$$
g(t)=\frac{\left(t-t_{1}\right) \ldots\left(t-t_{v}\right)}{\left(t-a_{1}\right)^{\kappa_{1}} \ldots\left(t-a_{r}\right)^{\kappa_{r}} \ldots\left(t-a_{p}\right)^{\alpha_{p}}} \cdot \frac{G(t)}{\left(t-a_{1}\right)^{q+1} \ldots\left(t-a_{r}\right)^{q} \ldots\left(t-a_{p}\right)^{q}},
$$

where $G$ is a polynomial of degree at most $s_{1}+\cdots+s_{p}-v$ if $Q_{n}$ is non-degenerate, and 
of degree at most $s_{1}+\cdots+s_{p}-1-v$ if $Q_{n}$ and $f$ (hence also $g$ ) are degenerate. By using the equalities $q p+r=n, \kappa_{1}+\cdots+\kappa_{p}=n+v-\mu$ (Theorem 2.6) we get $\operatorname{deg} G \leqq n+n+v-$ $\mu-1-v=(n=1)+(n-\mu)=n-1$ when $Q_{n}$ is non-degenerate, $\operatorname{deg} G \leqq n+n+v-\mu-1-1-$ $v=(n-1)+(n-1-\mu)=n-1$ when $Q_{n}$ and $f$ are degenerate. Thus we may write $f-F=$ $g=Q_{n} \cdot h$, where $h \in \mathscr{R}_{n-1}$. Consequently $\phi(f)-\phi(F)=\phi\left(Q_{n} \cdot h\right)=0$, and so

$$
\phi(f)=\sum_{i=1}^{v} f\left(t_{i}\right) \phi\left(L_{n, i}\right)=\sum_{i=1}^{v} \lambda_{n, i} f\left(t_{i}\right)
$$

Corollary 4.3. When $Q_{n}$ is non-singular the quadrature formula

$$
\phi(f)=\sum_{i=1}^{v} \lambda_{n, i} f\left(t_{i}\right)
$$

is valid for $f \in \mathscr{R}(2 q+2, \ldots, q+1, \ldots, 2 q)$.

Proof. Follows immediately from Theorem 4.2.

Proposition 4.4. The constants $\lambda_{n, i}$ are positive.

Proof. Note that we may write

$$
L_{n, i}(t)=\frac{D_{n}(t)}{\left(t-a_{1}\right)^{\alpha_{1}} \ldots\left(t-a_{r}\right)^{\alpha_{r}-1} \ldots\left(t-a_{p}\right)^{\alpha_{p}}},
$$

where $\operatorname{deg} D_{n}=v-1$. It follows that

$$
L_{n, i}(t)^{2}-L_{n, i}(t)=\frac{E_{n}(t)}{\left(t-a_{1}\right)^{2 \kappa_{1}} \ldots\left(t-a_{r}\right)^{2 q} \ldots\left(t-a_{p}\right)^{2 \kappa_{p}}}
$$

where $\operatorname{deg} E_{n}=2 v-2=2\left(\kappa_{1}+\cdots+\kappa_{p}\right)-2(n-\mu)-2$ by Theorem 2.6. Thus $\operatorname{deg} E_{n}=$ $2 \kappa_{1}+\cdots+2 q+\cdots+2 \kappa_{p}-2(n-\mu)$, and consequently $L_{n, i}^{2}-L_{n, i}$ belongs to $\mathscr{R}\left(2 \kappa_{1}, \ldots, 2 q, \ldots, 2 \kappa_{p}\right)$, and to $\mathscr{R}^{0}\left(2 \kappa_{1}, \ldots, 2 q, \ldots, 2 \kappa_{p}\right)$ if $Q_{n}$ is degenerate. Since $2 \kappa_{1} \leqq$ $\kappa_{1}+q+1, \ldots, 2 q \leqq 2 q+1, \ldots, 2 \kappa_{p} \leqq \kappa_{p}+q$, we conclude from Theorem 4.2 that

$$
\phi\left(L_{n, i}^{2}-L_{n, i}\right)=\sum_{j=1}^{v}\left[L_{n, i}\left(t_{j}\right)^{2}-L_{n, i}\left(t_{j}\right)\right] \lambda_{n, j}=0,
$$

and consequently $\lambda_{n, i}=\phi\left(L_{n, i}\right)=\phi\left(L_{n, i}^{2}\right)>0$.

4.5. Let $Q_{n}(t, \tau)$ denote the quasi-orthogonal $R$-functions introduced in Section 2.8 . We recall the meaning of $t_{i}(\tau), \kappa_{i}(\tau)$, etc. We define fundamental $R$-functions correspond- 
ing to $Q_{n}(t, \tau)$ in analogy with $L_{n, i}(t)$, i.e.

$$
L_{n, i}(t, \tau)=\frac{Q_{n}(t, \tau) \cdot\left(t-a_{r}\right)}{\frac{\partial}{\partial t} Q_{n}\left(t_{i}(\tau), \tau\right)\left(t-t_{i}(\tau)\right)\left(t_{i}(\tau)-a_{r}\right)}
$$

We observe that $L_{n, i}(t, \tau) \in \mathscr{R}_{n-1}$, that $L_{n, i}\left(t_{j}(\tau), \tau\right)=\delta_{i j}$, and that $L_{n, i}(t, \tau)$ is degenerate iff $Q_{n}(t, \tau)$ is degenerate. Let $\lambda_{n, i}(\tau)$ denote $\phi\left(L_{n_{i}}(t, \tau)\right)$.

Theorem 4.6. When $Q_{n}(t, \tau)$ is non-degenerate, the quadrature formula

$$
\phi(f)=\sum_{i=1}^{v(\tau)} \lambda_{n, i}(\tau) f\left(t_{i}(\tau)\right)
$$

is valid for every $f \in \mathscr{R}\left(s_{1}(\tau), \ldots, s_{p}(\tau)\right)$, where $s_{1}(\tau)=\kappa_{1}(\tau)+q+1, \ldots, s_{r}(\tau)=2 q, \ldots, s_{p}(\tau)=$ $\kappa_{p}(\tau)+q$. When $Q_{n}(t, \tau)$ is degenerate, the same formula is valid for $f \in \mathscr{R}^{0}\left(s_{1}(\tau), \ldots, s_{p}(\tau)\right)$.

Proof. We define

$$
g(t, \tau)=f(t)-\sum_{i=1}^{v(\tau)} f\left(t_{i}(\tau)\right) L_{n, i}(t, \tau)
$$

As in the proof of Theorem 4.2 we verify that we may write

$$
g(t, \tau)=Q_{n}(t, \tau) \cdot \frac{G(t, \tau)}{\left(t-a_{1}\right)^{q+1} \ldots\left(t-a_{r}\right)^{q-1} \ldots\left(t-a_{p}\right)^{q}}
$$

where $G(t, \tau)$ is a polynomial of degree at most $n-2$ (note that $s_{r}(\tau)=2 q$, while $s_{r}=$ $2 q+1)$, both in the general case and when $Q_{n}(t, \tau)$ and $f(t)$ are degenerate. It follows that $g(t, \tau)=Q_{n}(t, \tau) \cdot h(t, \tau)$, where

$$
h(t, \tau)=\frac{G(t, \tau)}{\left(t-a_{1}\right)^{q+1} \ldots\left(t-a_{r}\right)^{q-1} \ldots\left(t-a_{p}\right)^{q}}
$$

belong to $\mathscr{R}_{n-1}$, and

$$
\frac{\left(t-a_{r-1}\right)}{\left(t-a_{r}\right)} h(t, \tau)=\frac{G(t, \tau)}{\left(t-a_{1}\right)^{q+1} \ldots\left(t-a_{r-1}\right)^{q}\left(t-a_{r}\right)^{q} \ldots\left(t-a_{p}\right)^{q}}
$$

belongs to $\mathscr{R}_{n-2}$. Consequently

$$
\phi(g(t, \tau))=\phi\left(Q_{n}(t, \tau) \cdot h(t, \tau)\right)=\phi\left(Q_{n}(t) \cdot h(t, \tau)\right)-\tau \phi\left(Q_{n-1}(t) \cdot \frac{\left(t-a_{r-1}\right)}{\left(t-a_{r}\right)} h(t, \tau)\right)=0
$$


Thus

$$
\phi(f)=\sum_{i=1}^{v(\tau)} f\left(t_{i}(\tau)\right) \phi\left(L_{n, i}(t, \tau)\right)=\sum_{i=1}^{v(\tau)} \lambda_{n, i}(\tau) f\left(t_{i}(\tau)\right)
$$

Proposition 4.7. The constants $\lambda_{n, i}(\tau)$ are positive.

Proof. As in the proof of Proposition 4.4 we find that $L_{n, i}(t, \tau)^{2}-L_{n, i}(t, \tau)$ belongs to $\mathscr{R}\left(2 \kappa_{1}(\tau), \ldots, 2 q, \ldots, 2 \kappa_{-}(\tau)\right)$, and to $\mathscr{R}^{0}\left(2 \kappa_{1}(\tau), \ldots, 2 q, \ldots, 2 \kappa_{p}(\tau)\right)$ if $Q_{n}(t, \tau)$ is degenerate. Consequently the quadrature formula (4.6) is valid for the function $f(t, \tau)=L_{n, i}(t, \tau)^{2}-$ $L_{n, i}(t, \tau)$, and the desired result follows as in the proof of Proposition 4.4.

\section{Moment problems}

5.1. Let $\left\{c_{n}^{(r)}: n=1,2, \ldots\right\}, r=1,2, \ldots, p$, be $p$ given sequences of real numbers. We seek necessary and sufficient conditions for the existence of at least one distribution function (i.e.: bounded real-valued non-decreasing function) $\psi$ with an infinite number of points of increase such that

$$
\int_{-\infty}^{\infty} d \psi(t)=1, \int_{-\infty}^{\infty} \frac{d \psi(t)}{\left(t-a_{r}\right)^{n}}=c_{n}^{(r)}, \quad r=1,2, \ldots, p, \quad n=1,2, \ldots
$$

We shall call this problem the extended Hamburger moment problem (EHMP). When $\psi$ is a solution of EHMP, the integral $\int_{-\infty}^{\infty} f(t) d \psi(t)$ is defined for every $R$-function $f$. We shall here treat the problem in the context of the theory of orthogonal $R$-functions.

5.2. The sequences $\left\{c_{n}^{(r)}\right\}$ determine a linear functional $\phi$ on $\mathscr{R}$ by way of the following definition:

$$
\phi\left(a_{0}+\sum_{r=1}^{p} \sum_{n=1}^{N_{r}} \frac{\alpha_{r n}}{\left(t-a_{r}\right)^{n}}\right)=\alpha_{0}+\sum_{r=1}^{p} \sum_{n=1}^{N_{r}} \alpha_{r n} c_{n}^{(r)}
$$

Obviously $\phi\left(\varphi_{r, n}\right)=c_{n}^{(r)}$, where

$$
\varphi_{r, n}(t)=\frac{1}{\left(t-a_{r}\right)^{n}}
$$

We want to determine a distribution function $\psi$ with an infinite number of points of increase which represents the functions $\phi$ on $\mathscr{R}$.

Proposition 5.3. If EHMP has a solution, then the sequences $\left\{c_{n}^{(r)}\right\}$ must be such that the functional $\phi$ is positive definite.

Proof. For every $R$-function $S$, the $R$-function $S^{2}$ has only a finite number of zeros. Therefore $\phi\left(S^{2}\right)=\int_{-\infty}^{\infty} S(t)^{2} d \psi(t)>0$, since $\psi$ has infinitely many points of increase. 
5.4. We have seen that positive definiteness of $\phi$ is a necessary condition for the existence of a solution of EHMP. We shall in the following assume that the sequences $\left\{c_{n}^{(r)}\right\}$ are such that $\phi$ is positive definite. Then the theory of orthogonal (and quasiorthogonal) $R$-functions presented in the preceding sections is valid.

Before proceeding we note that every distribution function $\psi$ for which all the moments $\int_{-\infty}^{\infty} \varphi_{r, m} d \psi(t)$ exist, gives rise to a functional $\Psi$ on $\mathscr{R}$ defined by $\Psi(f)=\int_{-\infty}^{\infty} f(t) d \psi(t)$.

Proposition 5.5. If $\psi$ is a distribution function for which all the moments $c_{n}^{(r)}=\int_{-\infty}^{\infty} \varphi_{r, n}(t) d \psi(t)$ exist, and if the functional $\Psi$ is positive definite, then $\psi$ has an infinite number of points of increase.

Proof. Assume that $\psi$ has only a finite set of points of increase $\left\{\tau_{1}, \ldots, \tau_{k}\right\}$. Clearly $\tau_{i} \neq a_{r}, i=1, \ldots, k, r=1, \ldots, p$. We define an $R$-function $S$, e.g. by

$$
S(t)=\frac{\left(t-\tau_{1}\right) \ldots\left(t-\tau_{k}\right)}{\left(t-a_{1}\right)^{k}}
$$

Then $\int_{-\infty}^{\infty} S(t)^{2} d \psi(t)=0$, since $\psi$ is a step function with jumps exactly at the points $\tau_{1} \ldots \tau_{k}$, where $S^{2}$ vanish. On the other hand $\int_{-\infty}^{\infty} S(t)^{2} d \psi(t)=\Psi\left(S^{2}\right)>0$, since $\Psi$ is assumed to be positive definite. This contradiction shows that $\psi$ must have infinitely many points of increase.

5.6. For fixed indices $r, m$ the function $\varphi_{r, m}$ belongs to $\mathscr{R}(0,0, \ldots, m, 0, \ldots, 0)$ (where $m$ is at the $r$ th place). We observe that $\mathscr{R}(0, \ldots, 0, m, 0, \ldots, 0) \subset \mathscr{R}\left(2 \kappa_{1}, \ldots, 2 q+1, \ldots, 2 \kappa_{p}\right)$ when $n$ is so large that $2 q_{n}+1 \geqq m$.

Hence for $q_{n} \geqq \frac{1}{2}(m-1)$ the quadrature formula (4.2) gives

$$
c_{m}^{(r)}=\phi\left(\varphi_{r, m}\right)=\sum_{j=1}^{v_{n}} \lambda_{n, j} \varphi_{r, m}\left(t_{j}^{(n)}\right)
$$

where $t_{j}^{(n)}, j=1, \ldots, v_{n}$ are the zeros of $Q_{n}$.

Furthermore

$$
1=\phi(1)=\sum_{j=1}^{v_{n}} \lambda_{n, j}
$$

when $Q_{n}$ is non-degenerate.

For every index $n$ we define the step function $\psi_{n}$ by

$$
\psi_{n}(t)=\sum\left\{\lambda_{n, j}: t_{j}^{(n)} \leqq t\right\}
$$

Then we may write formula (5.2) as

$$
\int_{-\infty}^{\infty} \varphi_{r, m}(t) d \psi_{n}(t)=c_{m}^{(r)}, \quad \text { for } \quad q_{n} \geqq \frac{1}{2}(m-1) .
$$


We observe that $\psi_{n}$ is non-negative and non-decreasing, since $\lambda_{n, j}>0$.

Furthermore

$$
\int_{-\infty}^{\infty} d \psi_{n}(t)=1
$$

when $Q_{n}$ is non-degenerate. This formula implies that $0 \leqq \psi_{n}(t) \leqq 1$ for all $t \in(-\infty, \infty)$ when $n$ is non-degenerate.

5.7. We recall Helly's selection theorems (see e.g. [4]):

(H1) Let $\left\{f_{n}: n=0,1, \ldots\right\}$ be a uniformly . bounded sequence of real-valued nondecreasing functions on an interval $[a, b]$ (possibly $[a, b]=(-\infty, \infty)$ ). Then there exists a subsequence $\left\{f_{n_{k}}: k=0,1,2 \ldots\right\}$ which converges to a (non-decreasing) function $f$ for every $t \in[a, b]$

(H2) Let a uniformly bounded sequence $\left\{g_{m}: m=0,1, \ldots\right\}$ of real-valued nondecreasing function on a compact interval $[a, b]$ converge to a function $f$ for every $t \in[a, b]$. Then

$$
\lim _{m \rightarrow \infty} \int_{a}^{b} h(t) d g_{m}(t)=\int_{a}^{b} h(t) d f(t),
$$

for every continuous function $h$ on $[a, b]$.

Proposition 5.8. The sequence $\left\{\psi_{n}: n=1,2, \ldots\right\}$ has at least one convergent subsequence.

Proof. We recall that there are infinitely many non-degenerate indices (Corollary 3.7). Let $\left\{n_{k}: k=1,2, \ldots\right\}$ be the sequence of non-degenerate indices. It follows from Section 5.5 that the sequence $\left\{\psi_{n_{k}}: k=1,2, \ldots\right\}$ is uniformly bounded and consists of nondecreasing functions on $(-\infty, \infty)$. Hence by $(\mathrm{H} 1)$ there exists a convergent subsequence.

Proposition 5.9. Let $\psi(t)$ be the limit on $(-\infty, \infty)$ of some convergent subsequence $\left\{\chi_{i}(t): i=1,2, \ldots\right\}$ of the sequence $\left\{\psi_{n_{k}}(t): n_{k}\right.$ is non-degenerate $\}$. Then $\psi$ is a solution of the EHMP.

Proof. Formula (5.5) implies that for fixed $r$ and $m$ we have

$$
c_{m}^{(r)}=\int_{-\infty}^{\infty} \frac{d \chi_{i}(t)}{\left(t-a_{r}\right)^{m}}
$$

for sufficiently large $i$. Theorem (H2) implies that

$$
\int_{a}^{b} \frac{d \psi(t)}{\left(t-a_{r}\right)^{m}}=\lim _{i \rightarrow \infty} \int_{a}^{b} \frac{d \chi_{i}(t)}{\left(t-a_{r}\right)^{m}}
$$


for every compact interval not containing $a_{r}$. From these facts follow, e.g. by an argument analogous to that of [10], that the integrals

$$
\int_{-\infty}^{\infty} \frac{d \psi(t)}{\left(t-a_{r}\right)^{m}}
$$

exist and that

$$
c_{m}^{(r)}=\int_{-\infty}^{\infty} \frac{d \psi(t)}{\left(t-a_{r}\right)^{m}}
$$

Similarly the integral $\int_{-\infty}^{\infty} d \psi(t)$ exists and equals 1 .

It follows by Proposition 5.5 that the distribution function $\psi$ solves the EHMP for the given sequences.

Theorem 5.10. The EHMP for the sequences $\left\{c_{n}^{(r)}\right\}$ has a solution iff the sequences are such that the functional $\phi$ is positive definite.

Proof. Follows immediately from Proposition 5.3, Section 5.4 and Propositions 5.8 and 5.9.

5.11. We may generalize the definition of the functions $\psi_{n}(t)$ as follows: For every index $n$ and every real $\tau$ we define the step function $\psi_{n}(t, \tau)$ by

$$
\psi_{n}(t, \tau)=\sum\left\{\lambda_{n, j}(\tau): t_{j}^{(m)}(\tau) \leqq t\right\}
$$

where $t_{j}^{(m)}(\tau), j=1, \ldots v_{n}(\tau)$, are the zeros of $Q_{n}(t, \tau)$. Then again $\psi_{n}(t, \tau)$ is non-negative and non-decreasing as a function of $t, 0 \leqq \psi_{n}(t, \tau) \leqq 1$ when $Q_{n}(t, \tau)$ is non-degenerate, and

$$
c_{m}^{(r)}=\int_{-\infty}^{\infty} \varphi_{r, m}(t) d \psi(t, \tau)=\int_{-\infty}^{\infty} \frac{d \psi_{n}(t, \tau)}{\left(t-a_{r}\right)^{m}}
$$

for sufficient large $n$. We recall that $Q_{n_{k}}(t, \tau)$ is non-degenerate for every value of $\tau$ except one.

The following result gives no extra information on the EHMP, but it may provide a larger family of solutions to the problem.

Theorem 5.12. Let $\psi(t)$ be the limit on $(-\infty, \infty)$ of some convergent subsequence $\left\{\chi_{i}(t): i=1,2, \ldots\right\}$ of a sequence $\left\{\psi_{n}\left(t, \tau_{n}\right): n=1,2, \ldots\right\}$, where $Q_{n}\left(t, \tau_{n}\right)$ is non-degenerate. Then $\psi$ is a solution of the EHMP for the given sequences $\left\{c_{n}^{(r)}\right\}$.

Proof. The sequence $\left\{\psi_{n}\left(t, \tau_{n}\right)\right\}$ is uniformly bounded, and so the arguments in the proof of Proposition 5.9 may be carried over to the present situation. 


\section{REFERENCES}

1. N. I. Akiezer, The Classical Moment Problem and some Related Questions in Analysis (Hafner Publishing Company, New York, 1965).

2. C. Brezinski, Padé-type Approximation and General Orthogonal Polynomials (Birkhäuser Verlag, Basel-Boston Stuttgart, 1980).

3. T. S. Chinara, Introduction to Orthogonal Polynomials (Mathematics and its applications series, Gordon, 1978).

4. G. Freud, Orthogonal Polynomials (Pergamon Press, New York, 1971).

5. H. Hamburger, Über eine Erweiterung des Stieltjesschen Momentproblems, Parts I, II, III, Math. Ann. 81 (1920), 235-319, 82 (1921), 120-164, 168-187.

6. William B. Jones and W. J. Thron, Orthogonal Laurent polynomials and Gaussian quadrature', Quantum Mechanics in Mathematics, Chemistry and Physics (Eds., K. Gustafson and W. P. Reinhardt, Plenum Publishing Corp., New York, 1981), 449-455.

7. William B. Jones and W. J. Thron, Survey of continued fractions methods of solving moment problems and related topics, Analytic Theory of Continued Fractions, Proceedings, Loen, Norway, 1981 (Lecture notes in mathematics, No. 932, Springer Verlag, Berlin, 1982), 4-37.

8. William B. Jones, Olav NuÅstad and W. J. Thron, Two-point Padé expansions for a family of analytic functions, J. Comp. Appl. Math. 9 (1983), 105-123.

9. William B. Jones, Olav Nuåstad and W. J. Thron, Continued fractions and strong Hamburger moment problems, Proc. London Math. Soc. (3) 47 (1983), 363-384.

10. William B. Jones, Olav NuÅstad and W. J. Thron, Orthogonal Laurent polynomials and the strong Hamburger moment problem, J. Math. Anal. App., to appear.

11. William B. Jones, W. J. Thron and H. WaAdeland, A strong Stieltjes moment problem, Trans. Amer. Math. Soc. (1980), 503-528.

12. Olav NuÁstad and W. J. Thron, The theory of sequences of orthogonal L-polynomials, Padé Approximants and Continued Fractions, (Eds., Haakon Waadeland and Hans Wallin), Det Kongelige Norske Videnskabers Selskab, Skrifter (1983) No. 1, 54-91.

13. Olav Nus̊stad and W. J. Thron, Unique solvability of the strong Hamburger moment problem, J. Austral. Math. Soc. (Series A) 39 (1985), 15 pages.

14. J. A. Shohat and J. D. Tamarkin, The Problem of Moments (Mathematical Surveys No. 1, Amer. Math. Soc., Providence, R.I., 1943).

15. T. J. Stieltues, Reschérches sur les fractions continues, Ann. Fac. Sci, Toulouse 8 (1984), J, 1-122; 9 (1898), A, 1-47; Oeuvres 2 402-566.

UNIVERSITY OF TRONDHEIM-NTH

N-7034 TRONDHEIM

NoRWAY 